\title{
Ethnicity, Social, and Clinical Risk Factors to Tooth Loss among Older Adults in the U.S., NHANES 2011-2018
}

\author{
Haeok Lee ${ }^{1, *}$, Deogwoon Kim $^{1}\left(\mathbb{D}\right.$, Andrew Jung ${ }^{2}$ and Wonjeong Chae ${ }^{3} \mathbb{D}$ \\ 1 Nursing Department, University of Massachusetts Boston, Robert and Donna Manning College of Nursing \\ and Health Sciences, Boston, MA 02125, USA; deogwoon.kim001@umb.edu \\ 2 College of Dentistry, New York University, New York, NY 10012, USA; aj1827@nyu.edu \\ 3 Office of Strategic Planning, Healthcare Policy and Strategy Task Force, Yonsei University Health System, \\ Seoul 03722, Korea; wjchae0816@yuhs.ac \\ * Correspondence: haeok.lee@umb.edu
}

Citation: Lee, H.; Kim, D.; Jung, A.; Chae, W. Ethnicity, Social, and Clinical Risk Factors to Tooth Loss among Older Adults in the U.S., NHANES 2011-2018. Int. J. Environ. Res. Public Health 2022, 19, 2382. https://doi.org/10.3390/ ijerph19042382

Academic Editor: Iole Vozza

Received: 5 January 2022

Accepted: 10 February 2022

Published: 18 February 2022

Publisher's Note: MDPI stays neutral with regard to jurisdictional claims in published maps and institutional affiliations.

Copyright: (C) 2022 by the authors. Licensee MDPI, Basel, Switzerland. This article is an open access article distributed under the terms and conditions of the Creative Commons Attribution (CC BY) license (https:// creativecommons.org/licenses/by/ $4.0 /)$.

\begin{abstract}
Background. Many older adults suffer from poor oral health, including tooth loss, and disparities among racial/ethnic and socially disadvantaged populations continue to exist. Methods. Data were obtained from the National Health and Nutrition Examination Survey among the adult population in the U.S. The prevalence of edentulism and multiple regression models were conducted on 15,821 adults, including Asians, Blacks, Hispanics, Whites, and others to assess the relationships between tooth loss and their predictors. Results. The prevalence of complete tooth loss increased with age from $0.7 \%$ for ages $20-44$ to $20.2 \%$ for ages 65 and over. There are disparities in complete tooth loss regarding race/ethnicity, with the highest percentages (9\%) among Whites and Blacks and the lowest percentages among Asians (3\%) and Hispanics (4\%). After adjusting for predictors, their impact on tooth loss was not consistent within racial/ethnic groups, as Asians had more tooth loss from Model $1(\beta=-1.974, p<0.0001)$ to Model $5(\beta=-1.1705, p<0.0001)$. Conclusion. Tooth loss was significantly higher among older adults and racial/ethnic groups even after controlling for other predictors among a nationally representative sample. The findings point to the fact that subgroup-tailored preventions are necessary.
\end{abstract}

Keywords: older adults; oral health; health disparities; ethnicity; dementia; social factors

\section{Introduction}

According to the World Population Prospects 2019, by 2050, 1 in 6 people in the world will be over the age of 65, up from 1 in 11 in 2019 [1]. There is an urgent call to respond to major changes in the demographic composition of the world population and health related to rapidly growing aging. Aging is one aspect of successful human history, as improved nutrition, hygiene, and other factors have contributed to increasing life spans worldwide. However, dementia and oral disease are major challenges in caring for older adult populations [2-6]. Most oral diseases result from complex interactions influenced by genetic, biological, socioeconomic and behavioral health factors [7-10]. Globally, commonly reported poor oral conditions associated with older individuals include tooth loss, dry mouth (xerostomia), and higher numbers of dental caries and periodontal disease [11,12]. Oral diseases are some of the most expensive diseases, accounting for $\$ 545$ billion in direct and indirect costs globally [13]. In the U.S., preventive dental costs can incur tremendous costs at $\$ 60.5$ billion of all healthcare spending, which is far greater than health spending on other disorders, including lung cancer, drug use disorders, and alcohol use in 2016 [14]. Studies provide evidence of an association between tooth loss and dementia, which was stronger for older adults $[6,15,16]$. Thus, the socioeconomic burden of oral disease is expected to increase substantially, as there is an increasing aging population globally.

According to the Centers for Disease Control and Prevention (CDC) report, one in six American older adults at an age of 65 and above suffers from tooth loss and are completely 
edentulous [17]. The prevalence of complete tooth loss among older adults aged 65 and above was $12.9 \%$, and Black older adults had a higher prevalence of complete tooth loss than White older adults (25.4\% vs. 10.9\%) [3]. Ethnic differences in missing teeth may be related to multiple factors from the individual, social, and health care system levels [10,18-21]. However, relative contributions of physical and sociocultural factors to ethnic differences in tooth loss have not been reported. There is scarce or little population-based data to clearly identify the unique oral health problems in rapidly growing racial/ethnic groupsAsian Americans and Hispanics in particular-who have linguistic and cultural barriers to accessing health care in the U.S.

Asians are the fastest growing racial/ethnic group in the U.S., with a population of more than 14 million as of 2010, which is projected to grow to nearly 36 million in 2060 [22]. However, in the CDC's "Healthy People 2020" report, as in most other national health reports, data of the prevalence of complete tooth loss were not provided on Asian Americans [23]. The most common notations in these reports regarding Asians are: "data have not been analyzed (DNA)", "data have not been collected (DNC)", and "data are statistically unreliable (DSU)". Thus, the lack of accurate population-based data from this ethnic group masks their health needs, because no data clearly attest to their unique health problems. In addition, it offers no defined baseline of the health status and health behavior of Asian Americans from which goals can be set and evaluated. Additionally, the absence of baseline data makes it almost impossible for grant funders, researchers, and practitioners to know where to target and how to reduce the health inequity gap [24].

Oral disease is common among older adults and involves tooth loss, and it is imperative to provide baseline data of tooth loss and its correlators reflecting race/ethnicity among adult populations. Therefore, this study is aimed at: (1) examining ethnic differences (Hispanic, non-Hispanic White, non-Hispanic Black, non-Hispanic Asian) in tooth loss among American young adults (20-44 years), middle-aged (45 to 64 years), and older adults (65 years and older); (2) examining the effects of bio-socio-culture-health behavior and health access factors on tooth loss in three different age groups; and (3) examining racial/ethnic differences in tooth loss while controlling important predictors (covaries).

\section{Materials and Methods}

\subsection{Data Source and Study Population}

The study extracted data from a nationally representative sample of adults, the National Health and Nutrition Examination Survey (NHANES). The NHANES was conducted by the National Center for Health Statistics of the CDC [25]. The survey participants were selected based on households, and they provided informed written consent for the survey. In the NHANES study, a household is defined as the first household member 18 years of age or older listed on the household member roster, who owns or rents the residence where members of the household reside. As for oral health measures, this includes the information collected during the home interview, the mobile examination center (MEC) examination, and specimens for lab analyses [26]. The home interview oral health questions covered topics including dental visit frequency, perceived oral health status, the receipt of preventive health information, oral pain, and periodontal disease. Details of survey and clinical oral examination methods have been previously published [27]. Data from the 2011 to 2018 NHANES were used, since NHANES has been oversampling Asian Americans in addition to traditionally oversampled groups, including Hispanic and non-Hispanic Blacks [25]. Though the data are available up to 2020, due to COVID-19, complete sets of survey data are only available until 2018. These analyses were based on de-identified public-use data. A total of 15,161 participants who were over 20 years old were selected for the study from the survey cycle of 2011-2018. Out of the total individuals, 48.7\% ( $n=7386)$ were males and $51.3 \%(n=7775)$ were females. The final analysis was restricted to the dependent variables of tooth loss and nine correlates of demographics, socioeconomics, health risks, oral health status, and health care access that are mostly used in epidemiological studies [8-10,28,29]. 


\subsection{Measures}

\subsubsection{Dependent Variable}

The number of missing teeth was used as the dependent variable. For the study, we reported it as two forms: categorical (four groups: none (0), 1 to 5 teeth, 6 to 31 teeth, all (32)) and continuous. Partial loss was measured as missing at least one tooth vs. no tooth loss. Complete loss was measured as complete tooth loss vs. none to any number of tooth loss. The variable was created by adding individual missing tooth counts if the individual did not have any missing teeth, and ranged from zero (full set of teeth) to 32 teeth.

\subsubsection{Correlated Variables}

We selected variables related to tooth loss based on a literature review [8-10] and classified them into five domains, including demographic, socioeconomic, health risk, oral health condition, and health care access.

The demographic domain included sex, age, and race/ethnicity. Race/ethnicity was assessed by self-reporting as non-Hispanic White, non-Hispanic Black, Hispanic, nonHispanic Asian and other. We will hence refer to non-Hispanic White, non-Hispanic Black, and non-Hispanic Asian as Asian, Black, and White, respectively. Age was considered in this study as a major explanatory variable with four categories $(20-44 ; 45-64$; and >65) and was included in all analyses.

Socioeconomic domains were education and income. Education included three categories (less than high school, high school, and above high school); and income was in four categories (up to $\$ 2099,2100$ to $5399 ; 5400$ to $8399 ; 8400$ ).

The health risk domain, related to oral health, included diabetes and smoking because of their known relationship with tooth loss $[8,10,30]$. Diabetes indicated a self-reported diagnosis of diabetes vs. non-diabetic. Smoking was categorized as every day, some days, and not at all.

The oral health domain included self-reported periodontal bone loss and previous gingival disease.

The health care access domain was measured with health care insurance and the last dental care visits. Dental visits included routine check-ups within the past 6 months or not. The health insurance variable was categorized as whether participants had private health coverage or not, since most people 65 years and older have Medicare.

\subsection{Data Analysis}

First, a descriptive data analysis assessed the distribution of all variables for all participants, and by tooth loss (one or more as an interval measure) and total tooth loss. The $t$-tests, ANOVA, and chi-squared tests were employed to investigate the differences in the number of missing teeth and variables. Then, multiple regression was performed to predict tooth loss among the sample, using all variables found to be significant in bivariate analysis, as well as variables with prior significance [8-10] using a generalized lineal model (GLM). The first model was adjusted for age, sex, and ethnicity. The second model was adjusted for education and income, and the third model was adjusted for the tooth lossrelated risk factors of smoking and diabetes. The fourth model was adjusted for a history of bone loss and gingival disease, as well as self-rated gingival health. The last model was additionally adjusted for private insurance and the last visit to a dentist. For multi-category variables, the reference group was chosen as the category generally associated with the lowest risk of tooth loss. For those selective variables, the variance inflation factors (VIFs) test was conducted to examine the multi-correlation between independent variables that all variables showed less than 10, which indicated no correlation. Results after analyses were presented with a beta-coefficient and adopted a significance level of 0.01 ( $p$-value $<0.01$ was considered statistically significant). All analyses were performed by SAS version 9.4 (SAS Institute, Cary, NC, USA). 


\section{Results}

The characteristics of the 15,161 participants are shown in Table 1. From the study sample of adults older than 20 years of age, 51\% were female and 39\% were non-Hispanic White, 23\% Hispanic, 22\% non-Hispanic Black, and 12\% non-Hispanic Asian. Overall, 52\% were born in the U.S., the majority $(82.5 \%)$ had health insurance, only $51 \%$ had private insurance, and 36.5\% reported utilizing dental services within 6 months. Related to health behavior risk factors, $14.6 \%(n=2218)$ had diabetes and $11.6 \%(n=1756)$ smoked every day. Twenty-two percent reported a history of gum disease, $13.8 \%$ a history of bone loss, and $38 \%$ reported that their gum health was very good.

The overall mean of missing teeth was 9.1 ( $\mathrm{SD} \pm 9.0$ ); the mean score for the age group of 20-44 was 4.1, for 45-64 it was 8.4, and for those aged 65 and higher it was 10.7. Eight percent had complete tooth loss, $45.2 \%$ had $1-5$ missing teeth, and $41.4 \%$ had $6-31$ missing. The prevalence of complete tooth loss increased with age from $0.7 \%$ for ages $20-44$ to $20.2 \%$ for ages 65 and over. There were disparities in complete tooth loss in race/ethnicity with a high percentage (9\%) among White and Black participants, and the lowest percentages among Asians (3\%) and Hispanics (4\%). Participants who had low education, low income, health risk factors, a dental condition, no private insurance, and no recent dentist visits were more likely to have fewer teeth compared with their reference groups.

Table 2 separately shows the association of selected predictors and the number of missing teeth for different age groups, along with the results of the Chi-square tests. Comparing the young adult population (aged 22-44 years) and the older adult population (>65 years), the results indicate that members of young adult groups have significantly fewer missing teeth than members of older adult groups. As per the race group, those who did not have missing teeth were in the order of Black (16.3\%), Hispanic $(12.9 \%)$, Asian $(12.4 \%)$, other race $(10.8 \%)$, and White $(6.0 \%)$ in the young adult group $(p<0.001)$. In comparison, the edentulous older adult group was in the order of other race $(34.4 \%)$, Black $(26.2 \%)$, White $(20.4 \%)$, Hispanic $(14.8 \%)$, and Asian $(11.8 \%)$, with statistically significant differences $(p<0.001)$.

Socioeconomic factors such as education and monthly income showed that rates of young adults with a full set of teeth were higher among those with low socioeconomic status (education level: below high school 14.8\% vs. above high school 10.0\%, $p<0.001$; income level: below \$2099: 11.9\% vs. above \$8400: 8.0\%, $p<0.001)$. However, among older adults, there were more edentulous subjects among those of low socioeconomic status (education level: below high school $31.1 \%$ vs. above high school 12.3\%, $p<0.001$; income level: below \$2099: $28.0 \%$ vs. above $\$ 8400$ : 5.9\%, $p<0.001$ ). Related to the health risk condition and healthcare access, those who had an unhealthy health condition and low healthcare access exhibited poor oral health, regardless of age group differences. Individuals with diabetes and a habit of smoking reported to be $23.7 \%$ and $67.7 \%$, respectively, with more edentulous subjects among older adults $(p<0.001)$. Moreover, those without private insurance $(23.4 \%)$ and who had last visited the dentist more than 6 months ago $(31.5 \%)$ had a higher prevalence of edentulousness among older adults $(p<0.001)$.

Additionally, the prevalence of complete tooth loss among older adults was significantly higher among those with low education levels, low income, with diabetes, and those who were currently smoking. Rates of complete tooth loss was also higher among those without private insurance and who visited the dentist less often; however, there was no significant difference observed in the prevalence of complete tooth loss between men and women. However, for the younger age group (20-44 years), there were substantially different associations observed between no tooth loss and sociodemographic factors. For instance, among the younger age group, the prevalence of no missing teeth was higher than those who were Black (16.3\%) compared with White $(6.0 \%)$. Similarly, the prevalence of no tooth loss was $14.8 \%$ among the group with less than a high-school education (14.8\%) compared with the group with more than a high-school education (10.0\%). 
Table 1. Selected sociodemographic characteristics and risk factors for tooth loss in adults, NHANES, 2011-2018 $(n=15,161)$

\begin{tabular}{|c|c|c|c|c|c|c|c|c|c|c|}
\hline & \multicolumn{2}{|c|}{ Total } & \multicolumn{8}{|c|}{ Missing Tooth } \\
\hline & $\mathbf{N}$ & $\%$ & $\begin{array}{c}\text { None (0) } \\
\%\end{array}$ & $\begin{array}{c}1 \sim 5 \\
\%\end{array}$ & $\begin{array}{c}6 \sim 31 \\
\%\end{array}$ & $\begin{array}{c}\text { All (32) } \\
\%\end{array}$ & $p$-Value ${ }^{\dagger}$ & Mean & S.E & $p$-Value $\ddagger$ \\
\hline Total & 15,161 & & 5.8 & 45.2 & 41.4 & 7.6 & $<0.001 "$ & 9.1 & 9.0 & $<0.001$ \\
\hline Age group & & & & & & & $<0.001$ & & & $<0.001$ \\
\hline $20 \sim 44$ & 5723 & 37.7 & 10.9 & 68.3 & 20.1 & 0.7 & & 4.4 & 4.1 & \\
\hline $45 \sim 64$ & 5688 & 37.5 & 3.9 & 39.4 & 50.5 & 6.2 & & 9.4 & 8.4 & \\
\hline$\geq 65$ & 3750 & 24.7 & 1.0 & 18.6 & 60.2 & 20.2 & & 16.1 & 10.7 & \\
\hline Male & 7386 & 48.7 & 61.8 & 48.7 & 46.5 & 51.1 & & 9.1 & 9.3 & \\
\hline Female & 7775 & 51.3 & 38.2 & 51.3 & 53.5 & 48.9 & & 9.2 & 8.8 & \\
\hline Race & & & & & & & $<0.001$ & & & $<0.001$ \\
\hline Hispanic & 3468 & 22.9 & 7.2 & 47.2 & 41.4 & 4.2 & & 8.0 & 8.0 & \\
\hline Non-Hispanic White & 5980 & 39.4 & 3.2 & 45.4 & 41.6 & 9.7 & & 9.9 & 9.5 & \\
\hline Non-Hispanic Black & 3300 & 21.8 & 7.4 & 37.2 & 46.4 & 9.1 & & 10.3 & 9.7 & \\
\hline Non-Hispanic Asian & 1867 & 12.3 & 8.8 & 54.8 & 33.4 & 3.1 & & 6.5 & 6.9 & \\
\hline $\begin{array}{c}\text { Other Race-Including } \\
\text { Multi-Racial }\end{array}$ & 546 & 3.6 & 6.8 & 44.7 & 37.5 & 11.0 & & 9.7 & 9.8 & \\
\hline Education level & & & & & & & $<0.001$ & & & $<0.001$ \\
\hline Below high school & 3229 & 21.3 & 6.0 & 31.7 & 47.6 & 14.6 & & 12.5 & 10.9 & \\
\hline Graduated high school & 3385 & 22.3 & 4.9 & 38.6 & 46.9 & 9.6 & & 10.5 & 9.7 & \\
\hline Above high school & 8547 & 56.4 & 6.1 & 52.8 & 37.0 & 4.1 & & 7.4 & 7.4 & \\
\hline Income (month) & & & & & & & $<0.001$ & & & $<0.001$ \\
\hline$\$ 0 \sim \$ 2099$ & 5490 & 36.2 & 5.1 & 35.0 & 47.2 & 12.7 & & 11.7 & 10.4 & \\
\hline$\$ 2100 \sim \$ 5399$ & 5713 & 37.7 & 6.1 & 45.2 & 42.4 & 6.3 & & 8.8 & 8.6 & \\
\hline$\$ 5400 \sim \$ 5399$ & 2008 & 13.2 & 6.4 & 55.3 & 35.4 & 2.8 & & 6.7 & 6.7 & \\
\hline$\$ 8400 \sim$ & 1950 & 12.9 & 6.4 & 63.4 & 28.7 & 1.6 & & 5.6 & 5.1 & \\
\hline Born in US & & & & & & & $<0.001$ & & & $<0.001$ \\
\hline Yes & 8521 & 56.2 & 5.8 & 54.7 & 53.1 & 11.0 & & 9.8 & 9.3 & \\
\hline No & 3783 & 25.0 & 10.4 & 57.8 & 46.5 & 5.5 & & 7.7 & 8.0 & \\
\hline Health insurance & & & & & & & $<0.001$ & & & $<0.001$ \\
\hline Yes & 12,509 & 82.5 & 5.1 & 44.3 & 42.2 & 8.3 & & 9.5 & 9.2 & \\
\hline No & 2652 & 17.5 & 9.1 & 49.2 & 37.7 & 4.0 & & 7.3 & 7.7 & \\
\hline
\end{tabular}


Table 1. Cont.

\begin{tabular}{|c|c|c|c|c|c|c|c|c|c|c|}
\hline & \multicolumn{2}{|c|}{ Total } & \multicolumn{8}{|c|}{ Missing Tooth } \\
\hline & $\mathbf{N}$ & $\%$ & $\begin{array}{c}\text { None (0) } \\
\%\end{array}$ & $\begin{array}{c}1 \sim 5 \\
\%\end{array}$ & $\begin{array}{c}6 \sim 31 \\
\%\end{array}$ & $\begin{array}{c}\text { All (32) } \\
\%\end{array}$ & $p$-Value ${ }^{\dagger}$ & Mean & S.E & $p$-Value $\ddagger$ \\
\hline Private insurance & & & & & & & $<0.001$ & & & $<0.001$ \\
\hline Yes & 7786 & 51.4 & 5.9 & 51.1 & 37.9 & 5.1 & & 7.9 & 7.9 & \\
\hline No & 7375 & 48.6 & 5.8 & 38.9 & 45.1 & 10.2 & & 10.5 & 9.9 & \\
\hline Diabetes & & & & & & & $<0.001$ & & & $<0.001$ \\
\hline Yes & 2218 & 14.6 & 2.8 & 26.5 & 55.5 & 15.2 & & 13.6 & 10.5 & \\
\hline No & 12,943 & 85.4 & 6.4 & 48.4 & 39.0 & 6.3 & & 9.4 & 8.5 & \\
\hline Current smoking status & & & & & & & $<0.001$ & & & $<0.001$ \\
\hline Every day & 1756 & 11.6 & 4.2 & 33.0 & 49.5 & 13.2 & & 12.1 & 10.4 & \\
\hline Some days & 410 & 2.7 & 7.3 & 46.8 & 39.5 & 6.3 & & 8.4 & 8.6 & \\
\hline Not at all & 12,995 & 85.7 & 6.0 & 46.7 & 40.4 & 6.8 & & 8.8 & 8.8 & \\
\hline Last visit to dentist & & & & & & & $<0.001$ & & & $<0.001$ \\
\hline 6 months or less & 5538 & 36.5 & 3.9 & 48.1 & 45.6 & 2.4 & & 7.8 & 6.9 & \\
\hline More than 6 months & 9623 & 63.5 & 7.0 & 43.5 & 39.0 & 10.5 & & 9.9 & 10.0 & \\
\hline History of bone loss (oral) & & & & & & & $<0.001$ & & & $<0.001$ \\
\hline History of gum disease & & & & & & & $<0.001$ & & & 0.001 \\
\hline Yes & 3377 & 22.3 & 4.1 & 41.1 & 51.2 & 3.6 & & 8.7 & 7.6 & \\
\hline No & 11,784 & 77.7 & 6.3 & 46.3 & 38.6 & 8.7 & & 9.3 & 9.4 & \\
\hline Self-rated gum health & & & & & & & $<0.001$ & & & $<0.001$ \\
\hline Very good & 5745 & 37.9 & 7.9 & 58.5 & 25.5 & 8.0 & & 7.4 & 9.5 & \\
\hline Good & 4693 & 31.0 & 5.4 & 42.9 & 42.1 & 9.6 & & 9.7 & 8.6 & \\
\hline Poor & 4723 & 31.2 & 3.7 & 31.1 & 60.1 & 5.0 & & 10.7 & 8.7 & \\
\hline
\end{tabular}


Table 2. Prevalence of tooth loss by sociodemographic characteristics and risk factors by age $(n=15,161)$.

\begin{tabular}{|c|c|c|c|c|c|c|c|c|c|c|c|c|c|c|c|}
\hline & \multicolumn{5}{|c|}{ 20-44 years, Number of Missing Teeth $(n=5723)$} & \multicolumn{5}{|c|}{ 45-64 years, Number of Missing Teeth $(n=5688)$} & \multicolumn{5}{|c|}{$\geq 65$ years, Number of Missing Teeth $(n=3750)$} \\
\hline & Total & $\begin{array}{c}\text { None (0) } \\
\%\end{array}$ & $\begin{array}{c}1 \sim 5 \\
\%\end{array}$ & $\begin{array}{c}6 \sim 31 \\
\%\end{array}$ & $\begin{array}{c}\text { All (32) } \\
\%\end{array}$ & Total & $\begin{array}{c}\text { None (0) } \\
\%\end{array}$ & $\begin{array}{c}1 \sim 5 \\
\%\end{array}$ & $\begin{array}{c}6 \sim 31 \\
\%\end{array}$ & $\begin{array}{c}\text { All (32) } \\
\%\end{array}$ & Total & $\begin{array}{c}\text { None }(0) \\
\%\end{array}$ & $\begin{array}{c}1 \sim 5 \\
\%\end{array}$ & $\begin{array}{c}6 \sim 31 \\
\%\end{array}$ & $\begin{array}{c}\text { All (32) } \\
\%\end{array}$ \\
\hline & 5723 & 10.9 & 68.3 & 20.1 & 0.7 & 5688 & 3.9 & 39.4 & 50.5 & 6.2 & 3750 & 1.0 & 18.6 & 60.2 & 20.2 \\
\hline Male & 2721 & 14.4 & 67.5 & 17.2 & 0.8 & 2776 & 4.8 & 41.1 & 47.9 & 6.2 & 1889 & 1.2 & 18.7 & 59.4 & 20.7 \\
\hline Female & 3002 & 7.8 & 69.0 & 22.7 & 0.6 & 2912 & 3.1 & 37.8 & 53.1 & 6.1 & 1861 & 0.9 & 18.5 & 61.0 & 19.7 \\
\hline \multicolumn{16}{|l|}{ Race } \\
\hline Hispanic & 1409 & 12.9 & 69.0 & 17.9 & 0.2 & 1368 & 4.2 & 41.0 & 51.8 & 3.1 & 691 & 1.3 & 15.1 & 68.9 & 14.8 \\
\hline Non-Hispanic White & 2060 & 6.0 & 69.5 & 23.1 & 1.5 & 2012 & 2.7 & 42.3 & 46.8 & 8.1 & 1908 & 0.8 & 22.7 & 56.1 & 20.4 \\
\hline Non-Hispanic Black & 1179 & 16.3 & 62.8 & 20.7 & 0.3 & 1370 & 3.2 & 29.8 & 59.6 & 7.4 & 751 & 0.9 & 10.4 & 62.5 & 26.2 \\
\hline Non-Hispanic Asian & 806 & 12.4 & 72.2 & 15.3 & 0.1 & 757 & 7.5 & 48.1 & 41.7 & 2.6 & 304 & 2.3 & 25.3 & 60.5 & 11.8 \\
\hline $\begin{array}{c}\text { Other Race-Including } \\
\text { Multi-Racial }\end{array}$ & 269 & 10.8 & 67.7 & 20.4 & 1.1 & 181 & 4.4 & 31.5 & 50.8 & 13.3 & 96 & 0.0 & 5.2 & 60.4 & 34.4 \\
\hline \multicolumn{16}{|l|}{ Education level } \\
\hline Below high school & 946 & 14.8 & 58.0 & 25.5 & 1.7 & 1235 & 3.7 & 30.5 & 55.1 & 10.6 & 1048 & 0.9 & 9.4 & 58.6 & 31.1 \\
\hline Graduated high school & 1221 & 10.7 & 64.9 & 23.6 & 0.8 & 1288 & 2.3 & 31.4 & 58.0 & 8.3 & 876 & 0.6 & 12.8 & 63.0 & 23.6 \\
\hline Above high school & 3556 & 10.0 & 72.2 & 17.5 & 0.4 & 3165 & 4.6 & 46.2 & 45.7 & 3.5 & 1826 & 1.3 & 26.6 & 59.8 & 12.3 \\
\hline \multicolumn{16}{|l|}{ Income (month) } \\
\hline$\$ 0 \sim \$ 2099$ & 1813 & 11.9 & 63.6 & 22.9 & 1.6 & 2042 & 2.6 & 28.2 & 58.7 & 10.4 & 1635 & 0.7 & 11.6 & 59.7 & 28.0 \\
\hline$\$ 5400 \sim 55399$ & 871 & 9.6 & 73.4 & 16.8 & 0.2 & 758 & 4.6 & 47.8 & 45.1 & 2.5 & 379 & 2.6 & 29.0 & 58.8 & 9.5 \\
\hline$\$ 8400 \sim$ & 772 & 8.0 & 78.8 & 13.2 & 0.0 & 892 & 6.4 & 59.0 & 33.1 & 1.6 & 286 & 1.7 & 35.7 & 56.6 & 5.9 \\
\hline \multicolumn{16}{|l|}{ Private Insurance } \\
\hline Yes & 2976 & 9.9 & 72.3 & 17.6 & 0.2 & 3043 & 4.8 & 47.4 & 44.6 & 3.3 & 1767 & 1.1 & 21.6 & 60.7 & 16.6 \\
\hline No & 2747 & 12.1 & 63.9 & 22.8 & 1.3 & 2645 & 2.8 & 30.3 & 57.4 & 9.5 & 1983 & 0.9 & 15.9 & 59.8 & 23.4 \\
\hline \multicolumn{16}{|l|}{ Diabetes } \\
\hline Yes & 239 & 8.4 & 60.7 & 29.3 & 1.7 & 974 & 3.4 & 30.1 & 56.7 & 9.9 & 1005 & 0.9 & 14.9 & 60.5 & 23.7 \\
\hline No & 5484 & 11.1 & 68.6 & 19.7 & 0.7 & 4714 & 4.0 & 41.3 & 49.3 & 5.4 & 2745 & 1.1 & 19.9 & 60.1 & 18.9 \\
\hline \multicolumn{16}{|l|}{ Current smoking status } \\
\hline Every day & 704 & 9.5 & 55.7 & 31.3 & 3.6 & 804 & 0.7 & 21.0 & 64.2 & 14.1 & 248 & 0.4 & 7.7 & 54.0 & 37.9 \\
\hline Some days & 221 & 13.1 & 64.7 & 21.7 & 0.5 & 142 & 0.7 & 31.7 & 59.9 & 7.7 & 47 & 0.0 & 8.5 & 61.7 & 29.8 \\
\hline Not at all & 4798 & 11.0 & 70.3 & 18.4 & 0.3 & 4742 & 4.5 & 42.8 & 48.0 & 4.8 & 3455 & 1.1 & 19.5 & 60.6 & 18.8 \\
\hline \multicolumn{16}{|l|}{ Last visit to dentist } \\
\hline 6 months or less & 1602 & 6.1 & 68.2 & 25.0 & 0.7 & 2358 & 4.1 & 47.2 & 46.5 & 2.1 & 1578 & 1.3 & 28.9 & 65.2 & 4.6 \\
\hline More than 6 months & 4121 & 12.8 & 68.3 & 18.2 & 0.7 & 3330 & 3.7 & 33.9 & 53.4 & 9.0 & 2172 & 0.8 & 11.1 & 56.6 & 31.5 \\
\hline
\end{tabular}


Table 2. Cont.

\begin{tabular}{|c|c|c|c|c|c|c|c|c|c|c|c|c|c|c|c|}
\hline & \multicolumn{5}{|c|}{ 20-44 years, Number of Missing Teeth $(n=5723)$} & \multicolumn{5}{|c|}{ 45-64 years, Number of Missing Teeth $(n=5688)$} & \multicolumn{5}{|c|}{$\geq 65$ years, Number of Missing Teeth $(n=3750)$} \\
\hline & Total & $\begin{array}{c}\text { None (0) } \\
\%\end{array}$ & $\begin{array}{c}1 \sim 5 \\
\%\end{array}$ & $\begin{array}{c}6 \sim 31 \\
\%\end{array}$ & $\begin{array}{c}\text { All (32) } \\
\%\end{array}$ & Total & $\begin{array}{c}\text { None (0) } \\
\%\end{array}$ & $\begin{array}{c}1 \sim 5 \\
\%\end{array}$ & $\begin{array}{c}6 \sim 31 \\
\%\end{array}$ & $\begin{array}{c}\text { All (32) } \\
\%\end{array}$ & Total & $\begin{array}{c}\text { None (0) } \\
\%\end{array}$ & $\begin{array}{c}1 \sim 5 \\
\%\end{array}$ & $\begin{array}{c}6 \sim 31 \\
\%\end{array}$ & $\begin{array}{c}\text { All (32) } \\
\%\end{array}$ \\
\hline & 5723 & 10.9 & 68.3 & 20.1 & 0.7 & 5688 & 3.9 & 39.4 & 50.5 & 6.2 & 3750 & 1.0 & 18.6 & 60.2 & 20.2 \\
\hline \multicolumn{16}{|c|}{$\begin{array}{l}\text { History of bone loss } \\
\text { (oral) }\end{array}$} \\
\hline Yes & 343 & 6.7 & 49.3 & 39.9 & 4.1 & 1005 & 1.8 & 26.5 & 63.5 & 8.3 & 744 & 0.3 & 15.7 & 67.9 & 16.1 \\
\hline No & 5380 & 11.2 & 69.5 & 18.8 & 0.5 & 4683 & 4.3 & 42.2 & 47.8 & 5.7 & 3006 & 1.2 & 19.3 & 58.3 & 21.2 \\
\hline \multicolumn{16}{|c|}{ History of gum disease } \\
\hline No & 4778 & 11.5 & 68.9 & 18.8 & 0.7 & 4143 & 4.0 & 39.9 & 48.8 & 7.3 & 2863 & 1.0 & 17.9 & 57.0 & 24.0 \\
\hline
\end{tabular}

Univariate analysis was used to test the differences of distribution of tooth loss within the age group. The Chi-square test was to test the difference in distribution of tooth loss by gender race, socioeconomic status, healthcare access, and health conditions and health behavior. All differences were significant $(p<0.001)$, except for sex in the $\geq 65$-year age group $(p=0.627)$ and health insurance in the $\geq 65$-year age group $(p=0.474)$. Bold shows its category. 
The results of multivariable linear regression analysis to predict the mean of missing tooth (continuous measure) and the simultaneous association of predictors and outcome variables are shown in Table 3. The first model for tooth loss was statistically significant $\left(\mathrm{R}^{2}=0.264 ; p<0.001\right)$, and all other models are shown to be statistically significant. The final model for eight predictors of tooth loss was significant $\left(R^{2}=0.352 ; p<0.001\right)$. Model 1 shows that the older age variable had the most substantial effect on tooth loss $(\geq 65$ : $\beta=11.60, p<0.001)$, and being Black $(\beta=1.05, p<0.001)$ was positively associated with tooth loss, while being Hispanic and Asian was negatively associated with tooth loss. In Model 2, the effect of ethnicity on tooth loss was changed with the inclusion of the economic variables, as Hispanics had less tooth loss, while Blacks and Asians had more tooth loss, but it remained almost unchanged for Blacks after the inclusion of health risks, clinical dental risks, and health care access. After adjusting for predictors, the effect of compounding variables on tooth loss are not consistent within racial/ethnic groups, as Asians had more tooth loss from Model $1(\beta=-1.97, p<0.001)$ to Model $5(\beta=-1.170, p<0.001)$, while Blacks (model 1: $\beta=1.05, p<0.001$ to model 5: $\beta=0.24, p=0.128$ ) and Hispanics (Model $1 \beta=-0.86, p<0.001$ to model $5 \beta=-1.99, p<0.001)$ had less tooth loss. In the full model (Model 5), older age, low education, and current smoking status are the three most important determinants of tooth loss, followed by ethnicity, low income, history of bone loss, and no recent dentist visits. Being male, Hispanic, and Asian, and having a history of gingival disease negatively predicted missing teeth. In addition, multivariate analyses with interactions on age group and income are presented in Supplementary Tables S1 and S2. Related to the age group, it showed the differences in oral health by ethnicity group was a similar trend in the young adult population, while it showed different trends in the older population. As per the income, regardless of ethnicity, individuals had poor oral health when they also had a lower income level. 
Table 3. Stepwise regression analysis of tooth loss for sociodemographic characteristics and risk factors among adults in the U.S., NHANES, 2011-2018 $(n=15,161)$.

\begin{tabular}{|c|c|c|c|c|c|c|c|c|c|c|c|c|c|c|c|}
\hline & \multicolumn{3}{|c|}{ Model 1} & \multicolumn{3}{|c|}{ Model 2} & \multicolumn{3}{|c|}{ Model 3} & \multicolumn{3}{|c|}{ Model 4} & \multicolumn{3}{|c|}{ Model 5} \\
\hline & $\beta$ & S.E. & $p$-Value & $\beta$ & S.E. & $p$-Value & $\beta$ & S.E. & $p$-Value & $\beta$ & S.E. & $p$-Value & $\beta$ & S.E. & $p$-Value \\
\hline \multicolumn{16}{|l|}{ Age group } \\
\hline $20 \sim 44$ & Ref. & & & Ref. & & & Ref. & & & Ref. & & & Ref. & & \\
\hline $45 \sim 64$ & 5.01 & 0.15 & $<0.001$ & 4.77 & 0.14 & $<0.001$ & 4.50 & 0.14 & $<0.001$ & 4.40 & 0.14 & $<0.001$ & 4.69 & 0.14 & $<0.001$ \\
\hline$\geq 65$ & 11.60 & 0.16 & $<0.001$ & 10.75 & 0.16 & $<0.001$ & 10.61 & 0.16 & $<0.001$ & 10.41 & 0.17 & $<0.001$ & 10.77 & 0.17 & $<0.001$ \\
\hline \multicolumn{16}{|l|}{ Sex } \\
\hline Male & -0.28 & 0.13 & 0.027 & -0.35 & 0.12 & 0.004 & -0.50 & 0.12 & $<0.001$ & -0.48 & 0.12 & $<0.001$ & -0.60 & 0.12 & $<0.001$ \\
\hline Female & Ref. & & & Ref. & & & Ref. & & & Ref. & & & Ref. & & \\
\hline \multicolumn{16}{|l|}{ Race } \\
\hline Hispanic & -0.86 & 0.17 & $<0.001$ & -2.18 & 0.17 & $<0.001$ & -1.94 & 0.17 & $<0.001$ & -1.82 & 0.17 & $<0.001$ & -1.99 & 0.17 & $<0.001$ \\
\hline Non-Hispanic White & Ref. & & & Ref. & & & Ref. & & & Ref. & & & Ref. & & \\
\hline Non-Hispanic Black & 1.05 & 0.17 & $<0.001$ & 0.45 & 0.16 & 0.006 & 0.42 & 0.16 & 0.010 & 0.47 & 0.16 & 0.003 & 0.24 & 0.16 & 0.128 \\
\hline Non-Hispanic Asian & -1.98 & 0.21 & $<0.001$ & -1.43 & 0.20 & $<0.001$ & -1.21 & 0.20 & $<0.001$ & -1.09 & 0.20 & $<0.001$ & -1.17 & 0.20 & $<0.001$ \\
\hline Other & & & & & & & & & & & & & & & \\
\hline Race-Including & 1.49 & 0.35 & $<0.001$ & 1.23 & 0.34 & $<0.001$ & 1.17 & 0.33 & $<0.001$ & 1.15 & 0.33 & 0.001 & 0.99 & 0.33 & 0.002 \\
\hline Multi-Racial & & & & & & & & & & & & & & & \\
\hline Graduated high & & & & 1.94 & 0.16 & $<0.001$ & 1.78 & 0.16 & $<0.001$ & 1.77 & 0.15 & $<0.001$ & 1.59 & 0.15 & $<0.001$ \\
\hline Above high school & & & & Ref. & & & Ref. & & & Ref. & & & Ref. & & \\
\hline \multicolumn{16}{|l|}{ Income (month) } \\
\hline$\$ 0 \sim \$ 2099$ & & & & 3.53 & 0.21 & $<0.001$ & 3.06 & 0.21 & $<0.001$ & 3.03 & 0.21 & $<0.001$ & 2.29 & 0.22 & $<0.001$ \\
\hline$\$ 2100 \sim \$ 5399$ & & & & 1.85 & 0.20 & $<0.001$ & 1.61 & 0.20 & $<0.001$ & 1.59 & 0.20 & $<0.001$ & 1.12 & 0.20 & $<0.001$ \\
\hline$\$ 5400 \sim \$ 5399$ & & & & 0.91 & 0.24 & $<0.001$ & 0.80 & 0.24 & 0.001 & 0.81 & 0.23 & 0.001 & 0.63 & 0.23 & 0.007 \\
\hline$\$ 8400 \sim$ & & & & Ref. & & & Ref. & & & Ref. & & & Ref. & & \\
\hline \multicolumn{16}{|l|}{ Diabetes } \\
\hline Yes & & & & & & & 1.89 & 0.18 & $<0.001$ & 1.85 & 0.18 & $<0.001$ & 1.76 & 0.17 & $<0.001$ \\
\hline No & & & & & & & Ref. & & & Ref. & & & Ref. & & \\
\hline \multicolumn{16}{|l|}{$\begin{array}{c}\text { Current smoking } \\
\text { status }\end{array}$} \\
\hline Yes & & & & & & & 2.97 & 0.19 & $<0.001$ & 2.87 & 0.19 & $<0.001$ & 2.69 & 0.19 & $<0.001$ \\
\hline No & & & & & & & Ref. & & & Ref. & & & Ref. & & \\
\hline
\end{tabular}


Table 3. Cont.

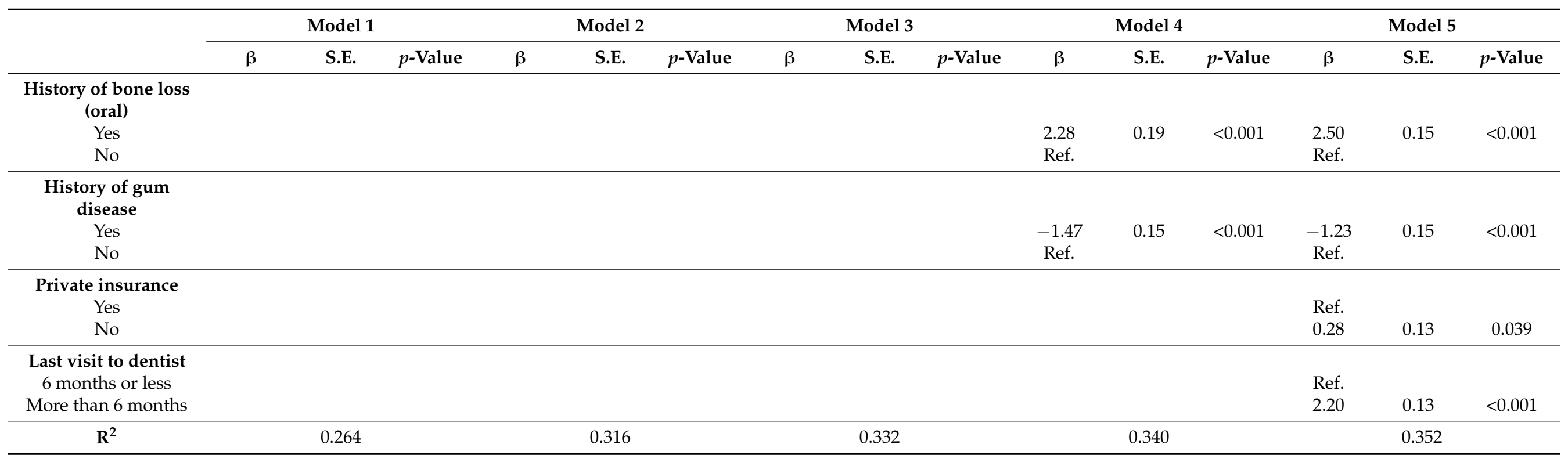

Bold shows its category. 


\section{Discussion}

The present study provided the most detailed descriptions of oral health status measured with tooth loss by age and race/ethnicity among adult populations in the U.S. Eliminating health disparities is the primary goal of Healthy People 2020, and the availability of reliable data is imperative to move toward health equity and achieve this goal. However, in Healthy People 2020, on the CDC website [23], it states, "OH-4.2: Adults with complete tooth loss (percent, 65-74 years)", and there are no specific objective data for Asian and Hispanic. Thence, the findings of this study will fill the data disparities gap to provide the first report on the prevalence of complete tooth loss among Asian (11.8\%) and Hispanic (14.8\%) ethnic minorities based on recent population data from the National Health and Nutrition Examination Survey, from 2011 to 2019. Our findings reveal that, in general, over the last two decades, edentulism declined from $12 \%$ to $7.6 \%$ for all groups (20 and older), especially among Whites from $18 \%$ to $9.7 \%$; however, there were no changes observed among Blacks (10\% to $9.1 \%$ ) and Hispanics (5\% to 4.2\%) from 1988-1994 NHANES [31] to 2011-2018 NHANES in the U.S. Our findings on the frequency of complete tooth loss among older Black and White adults $(26.2 \%$ and $20.4 \%)$ were higher than the CDC Healthy People 2020 report (21.1\% and 10.8\%) [23], and this finding is counterintuitive, as the age range in our study was 65 and higher, while CDC included only those aged between 65 and 74 years. The study demonstrated that women had higher rates of tooth loss and lower numbers of full sets of teeth than men, and this result is in line with studies that have observed that women present with higher rates of tooth loss [32,33].

Our findings on older adults are in line with a previous report that a higher prevalence of complete tooth loss was found among those who were Black, with low education levels and low incomes compared with the reference groups [20,32,34]. However, to the contrary, for the young adult group, low income and low education levels showed better oral health overall, with no missing teeth. This difference can be explained by that fact that the youngest (20-44) and oldest groups $(\geq 65)$ were exposed to very different life experiences than the current generation of the older adult population, and that the impact of socioeconomic factors accumulated to impact their health, including oral health. The data reported here are cross-sectional, so we can only imply the different effects of age, but cannot provide strong evidence for causation, and it cannot be ruled out that our findings are partially influenced by unobserved confounders. Hence, further studies are necessary to investigate the impact of inequality of SES and other predictors on oral health in different age groups.

Findings point out the fact that though sex differences in tooth loss are not significant in Model 1, it becomes significant for women when other predictors are taken into account. Studies report that men visit dentists less frequently compared to women, and that women are more likely to adhere to recommended treatment, even though women report more financial barriers than men [32,35-37]. This phenomenon of frequent dental visits and better adherence could contribute to the paradox of more dental extractions among women than men.

Our study demonstrates that oral health disparities are persistent across age groups and racial/ethnic groups, especially older adult populations in the U.S. Racial/ethnic differences remained even after controlling for other predictors. Being Hispanic and Asian was strongly negatively associated with tooth loss $(\beta=-2.03$ vs. $\beta=-1.20 ; p<0.001)$ after controlling for all other predictors. However, there was little variation in the number of missing teeth for multivariate adjusted analyses among Blacks, as the relationship between being Black and tooth loss was not significant once SES, physical risk factors, clinical dental condition, and health care access factors were included. On the contrary, after adding models with more predictors, Asians showed more tooth loss $(\beta=-1.98$ to $\beta=-1.20)$, while Hispanics had less tooth loss $(\beta=-0.86$ to $\beta=-2.30)$ from Models 1 to 5 . Our data suggest that the proposed covaries that are commonly used in epidemiology studies may not capture race-/ethnic group-specific indicators for oral health, especially related to missing teeth among diverse older adult populations. Additionally, it is possible that 
better oral health among Asian and Hispanic minorities in the U.S. could be attributable to the healthy migrant phenomenon [38]. The findings show that Hispanics are one ethnicity with lower tooth loss, but this does not change with the inclusion of all covaries, while the Asian population demonstrated the highest changes in tooth loss. Further studies are a necessity to explore the different directions of the influence of covaries or the selection bias of covaries and the sample.

\section{Strengths and Limitations}

This study has several strengths and limitations as well. The most important strength is a large sample size, which provides the power to evaluate the effect of covaries. The second is the NHANES survey methods. The inter-reliability of the oral health information, particularly the tooth count, was high between dental examiners (Kappa score: 0.96-1.00) [27]. Third, a multistage probability sampling design allowed NHANES to oversample racial/ethnic minorities and gain representative data about them. NHANES have oversampled Asian Americans since 2011 [39]. For data collection from participants with low English proficiency, NHANES uses nationwide translation interpreter services when language barriers are detected during eligibility screening. Consequently, this study showed that Asian Americans were successfully oversampled when compared to the census $(12.3 \%$ vs. $6 \%$ ) [40]. Lastly, the study cumulatively contains eight years of national survey data that tried to achieve external validity.

However, this study also has limitations. Although NHANES used diverse strategies to gain representative data from minorities, there still is a gap that may discourage minorities' participation or gain data that may not represent minorities with low English proficiency. For example, an advance letter that NHANES sent to selected households was prepared only in English and Spanish. Moreover, the NHANES questionnaire was translated only into English and Spanish, which means that the data quality from non-English speaking participants may have varied based on the interpreters who accompanied the data collectors. Additionally, the study design was a cross-sectional study, and there is no evidence to show causation from the results. The sociodemographic, health conditions and behaviors, healthcare access, and oral health (except for tooth loss) data were collected in a self-report format that could have had recall bias. This study had the number of missing teeth as its outcome, and missing wisdom teeth were also factored in as the definition of missing teeth in this study, applied conservatively. Although the study adjusted for confounding variables, there are potential confounding variables that are not included in this study; for example, among the study population, some missing teeth were wisdom teeth that were less likely to be related to poor oral health. In addition, due to the characteristics of NHANES, the study was not able to include clinical data for the adjustment.

\section{Conclusions}

Our study provided oral health outcomes, measured with tooth loss for four racial/ ethnic adult groups based on a nationally representative sample among adults [28]. As expected, the complete tooth loss was significantly high among older adults, that points to the fact that subgroup-tailored prevention, such as providing dentures and screening cognitive impairment for older adults, needs to be considered. Though racial/ethnicity differences remained even after controlling for all other predictors, the impact of predictors on each racial/ethnic group is varied when SES, physical, clinical dental, health behavior, and healthcare access factors are included in the model. Thus, its interpretation of the effect of the predictors of tooth loss on racial/ethnicity requires a caution, as it is difficult to drive causality and temporal issues from cross-sectional analysis. Longitudinal studies could be helpful to establish causal relationships. In addition, this study reveals several differences between men and women and different ethnic groups related to oral health, and highlights the need for further study to better understand these differences and develop prevention and education tailored to specific age, sex, and ethnicity to improve oral health among adults. 
Supplementary Materials: The following supporting information can be downloaded at: https: / / www.mdpi.com/article/10.3390/ijerph19042382/s1, Table S1: Results of multivariate analysis on ethnicity group and number of missing tooth by age group, Table S2: Results of multivariate analysis on ethnicity group and number of missing tooth by income.

Author Contributions: Conceptualization, H.L.; methodology, H.L., D.K. and W.C.; formal analysis, W.C., D.K. and H.L; resources, H.L.; data curation, W.C. and A.J.; writing-original draft preparation, H.L., W.C. and D.K.; writing-review and editing, A.J.; visualization, D.K.; supervision, H.L. funding acquisition, H.L. All authors have read and agreed to the published version of the manuscript.

Funding: National Institute on Aging: R56AG069130.

Institutional Review Board Statement: The study did not require ethical approval.

Informed Consent Statement: Not applicable.

Data Availability Statement: Data are available from National Centers for Health Statistics under the Centers for Disease Control and Prevention (https://www.cdc.gov/nchs/nhanes/index.htm, accessed date: 9 February 2022) for public use.

Conflicts of Interest: The authors declare no conflict of interest.

\section{References}

1. Department of Economic and Social Affairs. World Population Ageing 2019: Highlights; United Nations: New York, NY, USA, 2019.

2. Dye, B.; Thornton-Evans, G.; Li, X.; Iafolla, T. Dental Caries and Tooth Loss in Adults in the United States, 2011-2012; NCHS Data Brief: Hyattsville, MD, USA, 2015; p. 197.

3. Fleming, E.; Afful, J.; Griffin, S.O. Prevalence of tooth loss among older adults: United States, 2015-2018. NCHS Data Brief 2020, $368,1-8$.

4. Foley, N.C.; Affoo, R.H.; Siqueira, W.L.; Martin, R.E. A systematic review examining the oral health status of persons with dementia. DR Clin. Transl. Res. 2017, 2, 330-342. [CrossRef]

5. Patel, J.; Wallace, J.; Doshi, M.; Gadanya, M.; Yahya, I.B.; Roseman, J.; Srisilapanan, P. Oral health for healthy ageing. Lancet Healthy Longev. 2021, 2, e521-e527. [CrossRef]

6. Tai, C.; Chen, J.; Tseng, T.; Lin, Y.; Hsiao, Y.; Lee, M.; Yang, Y. Prediction of frailty and dementia using oral health impact profile from a population-based survey. Int. J. Environ. Res. Public Health 2020, 17, 1997. [CrossRef]

7. Beydoun, M.A.; Beydoun, H.A.; Hossain, S.; El-Hajj, Z.W.; Weiss, J.; Zonderman, A.B. Clinical and bacterial markers of periodontitis and their association with incident all-cause and Alzheimer's disease dementia in a large national survey. $J$. Alzheimer's Dis. 2020, 75, 157-172. [CrossRef] [PubMed]

8. Jiang, Y.; Okoro, C.A.; Oh, J.; Fuller, D.L. Sociodemographic and health-related risk factors associated with tooth loss among adults in Rhode Island. Prev. Chronic Dis. 2013, 10, E45. [CrossRef] [PubMed]

9. Lauritano, D.; Moreo, G.; Della Vella, F.; Di Stasio, D.; Carinci, F.; Lucchese, A.; Petruzzi, M. Oral health status and need for oral care in an aging population: A systematic review. Int. J. Environ. Res. Public Health 2019, 16, 4558. [CrossRef] [PubMed]

10. Wu, B.; Liang, J.; Plassman, B.L.; Remle, R.C.; Bai, L. Oral health among white, black, and Mexican-American elders: An examination of edentulism and dental caries. J. Public Health Dent. 2011, 71, 308-317. [CrossRef]

11. Petersen, P.E.; Yamamoto, T. Improving the oral health of older people: The approach of the WHO Global Oral Health Programme. Community Dent. Oral Epidemiol. 2005, 33, 81-92. [CrossRef]

12. World Health Organization. Oral Health in Ageing Societies: Integration of Oral Health and General Health; WHO Press: Geneva, Switzerland, 2006.

13. Righolt, A.J.; Jevdjevic, M.; Marcenes, W.; Listl, S. Global-, regional-, and country-level economic impacts of dental diseases in 2015. J. Dent. Res. 2018, 97, 501-507. [CrossRef]

14. Bolnick, H.J.; Bui, A.L.; Bulchis, A.; Chen, C.; Chapin, A.; Lomsadze, L.; Mokdad, A.H.; Millard, F.; Dieleman, J.L. Healthcare spending attributable to modifiable risk factors in the USA: An economic attribution analysis. Lancet Public Health 2020, 5, e525-e535. [CrossRef]

15. Yoo, J.J.; Yoon, J.H.; Kang, M.J.; Kim, M.; Oh, N. The effect of missing teeth on dementia in older people: A nationwide population-based cohort study in South Korea. BMC Oral Health 2019, 19, 61. [CrossRef]

16. Cerutti-Kopplin, D.; Feine, J.; Padilha, D.M.; de Souza, R.F.; Ahmadi, M.; Rompre, P.; Booij, L.; Emami, E. Tooth loss increases the risk of diminished cognitive function: A systematic review and meta-analysis. JDR Clin. Transl. Res. 2016, 1, 10-19. [CrossRef]

17. US Department of Health \& Human Services. Oral Health Surveillance Report: Trends in Dental Caries and Sealants, Tooth Retention, and Edentulism, United States, 1999-2004 to 2011-2016; Centers for Disease Control and Prevention: Atlanta, GA, USA, 2019.

18. Alos-Rullan, V. Households' age, country of birth, and marital status, stronger predictor variables than education in the prevalence of dental sealants, restorations, and caries among US children 5-19 years of age, NHANES 2005-2010. BMC Oral Health 2019, 19, 195. [CrossRef] 
19. Arora, G.; Mackay, D.F.; Conway, D.I.; Pell, J.P. Ethnic differences in oral health and use of dental services: Cross-sectional study using the 2009 Adult Dental Health Survey. BMC Oral Health 2016, 17, 1. [CrossRef]

20. Nazer, F.W.; Sabbah, W. Do socioeconomic conditions explain ethnic inequalities in tooth loss among US adults? Ethn. Dis. 2018, 28, 201-206. [CrossRef]

21. Sabbah, W.; Tsakos, G.; Sheiham, A.; Watt, R.G. The effects of income and education on ethnic differences in oral health: A study in US adults. J. Epidemiol. Community Health 2009, 63, 516-520. [CrossRef]

22. Pew Research Center. Asian Americans Are the Fastest-Growing Racial or Ethnic Group in the U.S. Available online: https:/ / www. pewresearch.org/fact-tank/2021/04/09/asian-americans-are-the-fastest-growing-racial-or-ethnic-group-in-the-u-s/ (accessed on 27 December 2021).

23. Office of Disease Prevention and Health Promotion. Oral Health. Available online: https://www.healthypeople.gov/2020 /topics-objectives/topic/oral-health/national-snapshot (accessed on 27 December 2021).

24. Lee, H.; Fitzpatrick, J.J.; Baik, S.Y. Why isn't evidence-based practice improving health care for minorities in the United States? Appl. Nurs. Res. 2013, 26, 263-268. [CrossRef]

25. National Center for Health Statistics. Overview: National Health and Nutrition Examination Survey. Available online: https: //wwwn.cdc.gov/nchs/nhanes/continuousnhanes/overview.aspx?BeginYear=2015 (accessed on 27 December 2021).

26. Centers for Disease Prevention and Control. National Health and Nutrition Examination Survey (NHANES): Oral Health Examiners Manual. Available online: https://wwwn.cdc.gov/nchs/data/nhanes/2017-2018/manuals/2018_Oral_Health_ Examiners_Manual.pdf (accessed on 30 December 2021).

27. Dye, B.A.; Afful, J.; Thornton-Evans, G.; Iafolla, T. Overview and quality assurance for the oral health component of the National Health and Nutrition Examination Survey (NHANES), 2011-2014. BMC Oral Health 2019, 19, 95. [CrossRef]

28. Helal, O.; Göstemeyer, G.; Krois, J.; Fawzy El Sayed, K.; Graetz, C.; Schwendicke, F. Predictors for tooth loss in periodontitis patients: Systematic review and meta-analysis. J. Clin. Periodontol. 2019, 46, 699-712. [CrossRef]

29. Tonetti, M.S.; Greenwell, H.; Kornman, K.S. Staging and grading of periodontitis: Framework and proposal of a new classification and case definition. J. Clin. Periodontol. 2018, 89, S159-S172. [CrossRef]

30. Zhang, Y.; Leveille, S.G.; Shi, L.; Camhi, S.M. Disparities in Preventive Oral Health Care and Periodontal Health Among Adults with Diabetes. Prev. Chronic Dis. 2021, 18, E47. [CrossRef] [PubMed]

31. Jimenez, M.; Dietrich, T.; Shih, M.; Li, Y.; Joshipura, K.J. Racial/ethnic variations in associations between socio-economic factors and tooth loss. Community Dent. Oral Epidemiol. 2009, 37, 267-275. [CrossRef] [PubMed]

32. Lipsky, M.S.; Su, S.; Crespo, C.J.; Hung, M. Men and oral health: A review of sex and gender differences. Am. J. Men's Health 2021, 15, 1-8. [CrossRef] [PubMed]

33. Meisel, P.; Reifenberger, J.; Haase, R.; Nauck, M.; Bandt, C.; Kocher, T. Women are periodontally healthier than men, but why don't they have more teeth than men? Menopause 2008, 15, 270-275. [CrossRef] [PubMed]

34. Steele, J.; Shen, J.; Tsakos, G.; Fuller, E.; Morris, S.; Watt, R.; Guarnizo-Herreño, C.; Wildman, J. The Interplay between socioeconomic inequalities and clinical oral health. J. Dent. Res. 2015, 94, 19-26. [CrossRef] [PubMed]

35. Ioannidou, E. The sex and gender intersection in chronic periodontitis. Front. Public Health 2017, 5, 189. [CrossRef]

36. Pinkhasov, R.M.; Wong, J.; Kashanian, J.; Lee, M.; Samadi, D.B.; Pinkhasov, M.M.; Shabsigh, R. Are men shortchanged on health? Perspective on health care utilization and health risk behavior in men and women in the United States. Int. J. Clin. Pract. 2010, 64, 475-487. [CrossRef]

37. Thompson, A.E.; Anisimowicz, Y.; Miedema, B.; Hogg, W.; Wodchis, W.P.; Aubrey-Bassler, K. The influence of gender and other patient characteristics on health care-seeking behaviour: A QUALICOPC study. BMC Fam. Pract. 2016, 17, 38. [CrossRef]

38. Sanders, A.E. A Latino advantage in oral health-related quality of life is modified by nativity status. Soc. Sci. Med. 2010, 71, 205-211. [CrossRef]

39. Paulose-Ram, R.; Burt, V.; Broitman, L.; Ahluwalia, N. Overview of Asian American data collection, release, and analysis: National Health and Nutrition Examination Survey 2011-2018. Am. J. Public Health 2017, 107, 916-921. [CrossRef]

40. United States Census Bureau. 2020 Census Illuminates Racial and Ethnic Composition of the Country. Available online: https:/ / www.census.gov/library/stories/2021/08/improved-race-ethnicity-measures-reveal-united-states-populationmuch-more-multiracial.html (accessed on 24 December 2021). 